\title{
Supplementation of Isomalto-oligosaccharide Reduced Colon Cancer-related Bacteria Enzymes and Fecal Toxicity in Nursing-home Residents
}

\author{
Hsiao-Ling Chen ${ }^{1,2, a, *}$ \\ ${ }^{1}$ Department of Food, Nutrition and Health Biotechnology, Asia University, 500, \\ Lioufeng Rd., Taichung city, Taiwan \\ ${ }^{2}$ Department of Medical Research, China Medical University Hospital, China Medical \\ University, Taichung city, Taiwan \\ ahlchen908@asia.edu.tw \\ ${ }^{*}$ Corresponding author
}

Keywords: Isomalto-oligosaccharide, Colorectal cancer, Nursing home $\beta$-glucuronidase, Mucinase, Bile acid, Cytotoxicity.

\begin{abstract}
This study investigated effects of 2-month isomalto-oligosaccharide (IMO) supplementation on fecal colonic bacterial enzymes, bile acid metabolites, and fecal toxicity in the nursing-home residents. Twelve nursing-home residents (mean age $85.2 \pm 1.9$ y. о.) participated in the placebo-controlled and diet-controlled study. This experiment consisted of a 1-month placebo, 2-month IMO (10 g indigestible oligosaccharide/d) and 1 month post (IMO withdrawal) period. Stool samples were collected for analyses of glucuronidase, mucinase, bile acids and cytotoxicity. The fecal glucuronidase and mucinase activity was significantly lowered by $32 \%$ and $22 \%$, respectively, with 1 month of IMO supplementation. The fecal glucuronidase and mucinase activity was furthermore reduced by $38 \%$ and $39 \%$ with 2 -month supplementation. Two-month IMO supplementation significantly decreased the fecal deoxycholic acid, a secondary bile acid related to colon cancer incidence by $57 \%$ and beneficially reduced the relative proportion of the secondary bile acid from $75.1 \%$ to $52.6 \%$. Overall, one-month IMO supplementation was sufficient to decrease the cytotoxicity of fecal water with 3 -h of co-incubation time. This human trial suggested that IMO (10 g/d) could effectively improve colonic ecology and reduce the colon cancer risk markers.
\end{abstract}

\section{Introduction}

Isomalto-oligosaccharide (IMO) is a well-known functional oligosaccharide which could be artificially derived from the starch [1-2]. IMO has been shown to exert beneficial gastrointestinal effects in men such as improving the defecation frequence and stool output in constipated elderly nursing-home residents [3-4], and acts as prebiotics to stimulate the growth of fecal bifidobacteria [4]. Therefore, it is considered as a functional dietary fiber and widely-used in the functional food to modulate the bowel function and improve the fecal flora profile. However, its role in the risk factors of colon carcinogenesis is not well-defined.

Colorectal cancer is the leading cause of cancer mortality in this and potentially future decades. Diet is considered as important modulatory factors [5]. For example, increased dietary fiber intake is associated with decreased the incidence of colorectal cancer [5]. Dietary fiber may reduce the colorectal cancer by improving the colonic ecology, such as cancer-associated bacterial enzymes, toxins and so on. In contrast, 
dietary fat is associated with increased cancer risk [6]. Bile acids are synthesized from dietary fat in the liver and secreted into the gastrointestinal tract. Part of the bile acids were reabsorbed to the liver via the enterohepatic circulation, and the rest enters the colon where they are metabolized into the carcinogenic secondary bile acids by bacteria [6]. In addition, bacterial $\beta$-glucuronidase converts the glycosides into carcinogenic a glycosides [5]; mucinase degrades mucus layer that may increase the exposure of the epithelium to toxins. Both enzymes increase the contact of colonic epithelium to toxic fecal ingredients. However, the role of IMO in these colonic bacterial activities and bile acid metabolism are not clear.

The aim of this study was to determine effects of IMO on human colorectal carcinogenic factors such as fecal enzyme activities, bile acid metabolites and fecal cyto-toxicity with a well-designed human trial.

\section{Subjects and Methods}

\section{Subjects}

Volunteers $(5 / 7$, male/female, $85.2 \pm 1.9$ y. o.) were recruited from a local nursing home [4]. The criteria for recruitment included willing to consume IMO for 2 months, a stable physiological condition, ability to consume a soft or blended diet, not bed-ridden, and no tobacco or antibiotic use. Routine medication maintained as usual.

\section{Experimental Design}

The experimental design of this human trial has been described previously [4]. In brief. a double-blind, placebo-controlled, diet-controlled study consisted of a 1-month placebo, 2-month IMO (IMO-1, IMO-2) and 1-month post (IMO withdrawal) period. Twenty-two $\mathrm{mL}$ of IMO containing $10 \mathrm{~g}$ indigestible oligosaccharide was given as an afternoon drink in the IMO period, while fructose was used as a placebo. IMO was withdrawn during the Post period. Diets during the trial were designed based on the nutrient requirement of individual subject as described previously [4]. Fecal samples were collected in the final week of each period, blended, lyophilized and prepared as fecal composite as described previously [4]. The study was proven by the Chung-Shan Medical University Ethics Committee and written informed consent was obtained from all subjects.

\section{Fecal $\beta$-glucuronidase and Mucinase Activities}

Fecal composite was extracted with PBS (0.1 M, pH 7.4) and centrifuged at 10,000 $\mathrm{g}$, $4^{\circ} \mathrm{C}$ for $10 \mathrm{~min}$ to obtain the supernatant for analysis. The $\beta$-glucuronidase activity was determined using the 4-nitrophenyl- $\beta$-D-glucuronide as substrate, and the release of 2-nitrophenol was measured at $405 \mathrm{~nm}$. Mucinase activity was determined using porcine gastric mucin as the substrate, and the release of reducing sugar was measured with the Nelson-Somogyi method [7]. The enzyme activity was expressed as IU/protein.

\section{Fecal Water Preparation and Bile Acid Analysis}

Fecal water, the supernatant obtained from feces, was prepared [8]. The primary bile acid (cholic and chenodeoxycholic acids) and the secondary bile acid (deoxycholic and lithocholic acids) were extracted and deriviatized with the method described previously [9]. Hyodeoxycgholic acid was used as an internal standard. Samples were dissolved in cyclohexane (Sigma Chemical Co., St. Louis, MO, USA) and analyzed using a gas chromatograph (GC-14B, Shimadzu Corp., Tokyo, Japan) fitted with a 
fused silica column (HP-5, $0.25 \mathrm{~mm} \times 0.25 \mu \mathrm{m} \times 60 \mathrm{~m}$, Agilent Technologies Inc., Santa Clara, CA, USA) and a flame ionization detector.

\section{Fecal Water Cytotoxicity}

The Caco-2 cells (Bioresource Collection and Research Center, Hsinchu, Taiwan) were used as a model of human colonocytes [8]. Cells were harvested at approximately $90 \%$ confluence and re-suspended in Hanks balanced salt solution (HBSS) at a concentration of $2 \times 10^{6}$ cells $/ \mathrm{mL}$ for further analysis. Cell viability was determined with trypan blue exclusion staining. Cytotoxicity is denoted as 1 - cell viability and each value was determined from three separate batch, each including three replicates.

\section{Statistical Analysis}

Values presented as mean \pm standard error of mean (SEM) were analyzed with SPSS version 17.0 for Windows (SPSS, Inc., Chicago, IL, USA). The data were analyzed with the General Linear Model repeated measures analysis of variance followed by pair-wise least significant difference (LSD) tests to compare the difference within four periods. Differences were considered significant at $P<0.05$.

\section{Results}

\section{Fecal Bacteria B-glucuronidase and Mucinase Activities}

The $\beta$-glucuronidase activity were significantly lower by $32 \%(P=0.038)$ and $38 \%(P$ $=0.024)$ in the IMO-1 and IMO-2 periods, respectively (Table 1). The mucinase activity was significantly $(22 \%, P<0.005)$ lower in the IMO-1, and this effect enlarged with longer supplementation to $39 \%(P<0.001)$.

Table 1. $\beta$-glucuronidase (IU/mg protein) and mucinase (IU/g protein) activities

\begin{tabular}{|l|c|c|c|c|}
\hline & Placebo & IMO-1 & IMO-2 & Post \\
\hline$\beta$-glucuronidase & $1.89 \pm 1.81^{\mathrm{b}}$ & $1.28 \pm 1.94^{\mathrm{a}}$ & $1.17 \pm 1.59^{\mathrm{a}}$ & $1.88 \pm 1.71^{\mathrm{b}}$ \\
\hline Mucinase & $5.80 \pm 1.87^{\mathrm{c}}$ & $4.51 \pm 1.50^{\mathrm{b}}$ & $3.56 \pm 1.31^{\mathrm{a}}$ & $4.65 \pm 1.55^{\mathrm{b}}$ \\
\hline
\end{tabular}

Values in a row not sharing the same superscript letters denote significant difference.

\section{Fecal Bile Acid Concentration and Profile}

Total fecal bile acid level did not differ among periods, but the bile acid composition was improved with IMO (Table 2). Fecal deoxycholic acid, a primary bile acid, were significantly $(P=0.017)$ increased with IMO throughout the study. This lead to the primary bile acids to total bile acid ratio beneficially increased as early as in the IMO-1 period, and this beneficial effect was more pronounced in the IMO-2 period. In line with that, the deoxycholic acid, a colon cancer-related bile acid, was significantly $(P=0.047)$ decreased in the IMO-2 period. Other bile acid level determined were not significantly modulated by the supplement. 
Table 2. Fecal bile acids level ( $\mu$ mole/g wet feces) and relative ratio (\%)

\begin{tabular}{|c|c|c|c|c|}
\hline & Placebo & IMO-1 & IMO-2 & Post \\
\hline \multicolumn{5}{|l|}{ Primary } \\
\hline $\mathrm{CA}$ & $0.07 \pm 0.01^{\mathrm{a}}$ & $0.12 \pm 0.03^{\mathrm{ab}}$ & $0.10 \pm 0.01^{b}$ & $0.13 \pm 0.03^{\mathrm{ab}}$ \\
\hline CDCA & $0.09 \pm 0.02$ & $0.11 \pm 0.03$ & $0.15 \pm 0.03$ & $0.11 \pm 0.02$ \\
\hline \multicolumn{5}{|l|}{ Secondary } \\
\hline DCA & $0.23 \pm 0.05^{\mathrm{a}}$ & $0.14 \pm 0.02^{\mathrm{ab}}$ & $0.10 \pm 0.03^{b}$ & $0.16 \pm 0.03^{\mathrm{ab}}$ \\
\hline LCA & $0.19 \pm 0.06$ & $0.14 \pm 0.03$ & $0.12 \pm 0.03$ & $0.14 \pm 0.04$ \\
\hline \multicolumn{5}{|c|}{ Relative ratio (\%) } \\
\hline Primary & $24.9 \pm 3.5^{\mathrm{a}}$ & $37.1 \pm 3.6^{b}$ & $47.4 \pm 4.1^{\mathrm{c}}$ & $39.1 \pm 5.1^{b}$ \\
\hline Secondary & $75.1 \pm 4.2^{\mathrm{a}}$ & $62.9 \pm 4.8^{b}$ & $52.6 \pm 4.0^{\mathrm{c}}$ & $60.9 \pm 4.2^{b}$ \\
\hline
\end{tabular}

Values in a row not sharing the same superscript letters denote significant difference. BA: bile acid; CA; cholic acid; CDCA; chenodeoxycholic acid; DCA; deoxycholic acid; LCA; lithocholic acid.

\section{Fecal Water Cytotoxicity}

The fecal water cytotoxicity was significantly lowered by $12-15 \%(P<0.02)$ with IMO supplementation.

Table 3. Fecal water cytotoxicity towards Caco-2 cells

\begin{tabular}{|c|c|c|c|c|}
\hline Hrs & Placebo & IMO-1 & IMO-2 & Post \\
\hline 1 & $0.10 \pm 0.02$ & $0.08 \pm 0.02$ & $0.12 \pm 0.02$ & $0.13 \pm 0.04$ \\
\hline 2 & $0.19 \pm 0.05$ & $0.15 \pm 0.03$ & $0.14 \pm 0.04$ & $0.16 \pm 0.04$ \\
\hline 3 & $0.22 \pm 0.04^{\mathrm{a}}$ & $0.13 \pm 0.03^{\mathrm{b}}$ & $0.11 \pm 0.03^{\mathrm{b}}$ & $0.14 \pm 0.04^{\mathrm{a}}$ \\
\hline
\end{tabular}

Values in a row not sharing the same superscript letters denote significant difference.

\section{Discussion}

The results of this study showed that supplementation of IMO into a typical Chinese low-fiber diet were able to reduce the risks of fecal metabolite related to colon cancer in elderly people. IMO reduced the bacteria glucuronidase, in agreement with our previous observation that dietary fibers reduced the glucuronidase activity in adult volunteers [5]. It is known that glucuronidase activity is different among bacteria. It is the highest in Escherichia coli and Clostridium but lowest in Bifidobacterium and Lactobacillus [10-11]. In addition, this study found that secondary bile acids, especially deoxycholic acid, were reduced with IMO. Bacteria 7 dehydroxylase, the rate-limiting enzyme converting the primary to secondary bile acid, appears to exist in clostridia and eubacteria, but was not detected in lactobacilli and bifidobacteria [12]. Therefore, the prebiotic effects of IMO modulate the colonic bacteria profile, which furthermore may improve the bacterial enzyme profile, and decreases the carcinogenic toxin formation. The lower toxic load of feces may beneficially reduce the cytotoxicity of fecal water towards Caco-2 cells which were observed in the IMO and post periods.

In conclusion, this study indicated that IMO supplementation beneficially reduced the fecal content toxicity and risk factors of colon carcinogenesis in long-term care subjects.

\section{Acknowledgments}

This study was supported by grant National Science Council 96-2320-B-040-031-MY3., Taiwan. Assistance from Dr. Yen, Chi-Hwa and Ms. Hsuan-Wen Yu are appreciated. 


\section{References}

[1] K. J. Duan, D. C. Sheu, C. T. Lin, Transglucosylation of a fungal alpha glucosidase. The enzyme properties and correlation of isomalto-oligosaccharide production, Ann. N. Y. Acad. Sci. 750 (1995) 325-328.

[2] A. Vetere, A. Gamini, C. Campa et al., Regiospecific transglycolytic synthesis and structural characterization of 6-O-alpha-glucopyranosyl- glucopyranose (isomaltose), Biochem. Biophys. Res. Commun. 274 (2000) 99-104.

[3] H.-L. Chen, Y.-H. Lu, J.-J. Lin et al., Effects of isomalto-oligosaccharides on bowel functions and indicators of nutritional status in constipated elderly men, J. Am. Coll. Nutr. 20 (2001) 44-49.

[4] C.-H. Yen, Y.-H. Tseng, Y.-W. Kuo et al., Long-term supplementation of isomalto-oligosaccharides improved colonic microflora profile, bowel function, and blood cholesterol levels in constipated elderly people-a placebo-controlled, diet-controlled trial, Nutrition 27(2011) 445-450.

[5] W.-T. Wu, H.-C. Cheng, H.-L. Chen, Ameliorative effects of konjac glucomannan on human faecal beta glucuronidase activity, secondary bile acid levels and faecal water toxicity towards Caco-2 cells, Br. J. Nutr. 105 (2011) 593-600.

[6] M. A. Rieger, A. Parlesak, B. L. Pool-Zobel et al., A diet high in fat and meat but low in dietary fibre increases the genotoxic potential of 'faecal water', Carcinogenesis 20 (1999) 2311-2316.

[7] N. Nelson, A photometric adaptation of the Somogyi method for the determination of glucose, J. Biol. Chem. 153 (1994) 75-378.

[8] S.-L. Yeh, M.-S. Lin, H.-L. Chen, Inhibitory effects of a soluble dietary fiber from Amorphophallus konjac on cytotoxicity and DNA damage induced by faecal water in Caco-2 cells, Planta Med. 73 (2007)1384-1388.

[9] F. Czubayko, B. Beumers, S. Lammsfuss et al., A simplified micro-method for quantification of faecal excretion of neutral and acidic sterols for outpatient studies in humans, J. Lipid Res. 32 (1991)1861-1867.

[10] O. Sreekumar, A. Hosono, Immediate effect of Lactobacillus acidophilus on the intestinal flora and faecal enzymes of rats and the in vitro inhibition of Escherichia coli in coculture, J. Dairy Sci. 83 (2000) 931-939.

[11] J. Nakamura, Y. Kubota, M. Miyaoka et al., Comparison of four microbial enzymes in Clostridia and Bacteroides isolated from human feces, Microbiol. Immunol. 46 (2002) 487-490.

[12] S. Hirano, R. Nakama, M. Tamaki et al., Isolation and characterization of thirteen intestinal microorganisms capable of 7 alpha-dehydroxylating bile acids, Appl. Environ. Microbiol. 41(1981) 737-745. 